\title{
Convergence of a New Modified Ishikawa Type Iteration for Common Fixed Points of Total Asymptotically Strict Pseudocontractive Semigroups
}

\author{
Yuanheng Wang and Chunjie Wang \\ Department of Mathematics, Zhejiang Normal University, Jinhua, Zhejiang 321004, China \\ Correspondence should be addressed to Yuanheng Wang; wangyuanheng@yahoo.com.cn
}

Received 28 April 2013; Revised 3 June 2013; Accepted 3 June 2013

Academic Editor: Abdellah Bnouhachem

Copyright (C) 2013 Y. Wang and C. Wang. This is an open access article distributed under the Creative Commons Attribution License, which permits unrestricted use, distribution, and reproduction in any medium, provided the original work is properly cited.

\begin{abstract}
The purpose of this paper is to give a new modified Ishikawa type iteration algorithm for common fixed points of total asymptotically strict pseudocontractive semigroups. Under the reduction of some conditions, both strong convergence and weak convergence of the iteration algorithm are proved in Banach spaces with new methods of proofs, respectively. The main results presented in this paper extend and improve the corresponding recent results of many others.
\end{abstract}

\section{Introduction}

Throughout this paper, we assume that $E$ is a real Banach space with the norm $\|\cdot\|, E^{*}$ the dual space of $E,\langle\cdot, \cdot\rangle$ the duality between $E$ and $E^{*}$, and $C$ a nonempty closed convex subset of $E$. $\mathbb{R}^{+}$denotes the set of nonnegative real numbers and $\mathbb{N}$ the natural number set. The mapping $J: E \rightarrow 2^{E^{*}}$ with

$$
J(x)=\left\{f^{*} \in E^{*}:\left\langle x, f^{*}\right\rangle=\|x\|^{2},\left\|f^{*}\right\|=\|x\|\right\}, \quad x \in E,
$$

is called the normalized duality mapping.

Let $T: C \rightarrow C$ be a nonlinear mapping. $F(T)$ denotes the set of the fixed points of $T$.

As we know, a mapping $T: C \rightarrow C$ is said to be pseudocontractive, if, for all $x, y \in C$, there exists $j(x-y) \in J(x-y)$, such that

$$
\langle T x-T y, j(x-y)\rangle \leq\|x-y\|^{2} .
$$

Variational inequalities introduced by Stampacchia in the early sixties have had a great impact and influence on the development of almost all branches of pure and applied sciences and have witnessed an explosive growth in theoretical advances, algorithmic development, and so forth.
Recently, some authors also studied the problem of finding the solution set of variational inequalities and the common element of the fixed point set for generalized nonexpansive mappings in the framework of real Hilbert spaces and Banach spaces. As is known to all, the variational inequality problem, nonlinear optimization problem, and fixed point problem are equivalent to each other under certain conditions.

In 2012, Chang et al. [1] introduced a more general class of pseudocontractive mappings and studied the methods for approximation of the split common fixed points.

Definition 1 (see [1]). (I) A mapping $T: C \rightarrow C$ is said to be $\left(\gamma, \mu_{n}, \xi_{n}, \phi\right)$-totally asymptotically strictly pseudocontractive, if there exist a constant $\gamma \in[0,1]$ and sequences $\left\{\mu_{n}\right\}$, $\left\{\xi_{n}\right\} \subset[0, \infty)$ with $\mu_{n} \rightarrow 0$ and $\xi_{n} \rightarrow 0$, such that, for all $x, y \in C$,

$$
\begin{aligned}
\left\|T^{n} x-T^{n} y\right\|^{2} \leq & \|x-y\|^{2}+\gamma\left\|\left(I-T^{n}\right) x-\left(I-T^{n}\right) y\right\|^{2} \\
& +\mu_{n} \phi(\|x-y\|)+\xi_{n}, \quad \forall n \geq 1,
\end{aligned}
$$

where $\phi:[0, \infty) \rightarrow[0, \infty)$ is continuous and a strict increasing function with $\phi(0)=0$.

(II) A mapping $T: C \rightarrow C$ is said to be $\left(\gamma, k_{n}\right)$ asymptotically strictly pseudocontractive, if there exist 
a constant $\gamma \in[0,1)$ and a sequence $k_{n} \subset[1, \infty)$ with $k_{n} \rightarrow 1$, such that

$$
\begin{aligned}
\left\|T^{n} x-T^{n} y\right\|^{2} \leq & k_{n}\|x-y\|^{2} \\
& +\gamma\left\|\left(I-T^{n}\right) x-\left(I-T^{n}\right) y\right\|^{2}, \quad \forall x, y \in C .
\end{aligned}
$$

Definition 2. (I) One-parameter family $\mathscr{T}:=\{T(t): C \rightarrow C$, $t \geq 0\}$ is said to be a pseudocontractive semigroup on $C$, if the following conditions are satisfied:

(a) $T(0) x=x$ for each $x \in C$;

(b) $T(t+s) x=T(t) T(s) x$ for any $t, s \in \mathbb{R}^{+}$and $x \in C$;

(c) the mapping $t \rightarrow T(t) x$ is continuous for any given $x \in C$;

(d) for any $t \geq 0, T(t)$ is pseudocontractive; that is, for any $x, y \in C$, there exists $j(x-y) \in J(x-y)$, such that

$$
\left\langle T^{n}(t) x-T^{n}(t) y, j(x-y)\right\rangle \leq\|x-y\|^{2} .
$$

(II) One-parameter family $\mathscr{T}:=\{T(t): C \rightarrow C, t \geq 0\}$ is said to be strict pseudocontractive semigroup on $C$, if the conditions (a)-(c) and the following condition (e) are satisfied.

(e) For any $x, y \in C$, there exist $j(x-y) \in J(x-y)$ and a bounded function $\eta:[0, \infty) \rightarrow[0, \infty)$, such that, for any $t \geq 0$,

$$
\begin{aligned}
& \langle T(t) x-T(t) y, j(x-y)\rangle \\
& \quad \leq\|x-y\|^{2}-\eta(t)\|(x-y)-(T(t) x-T(t) y)\|^{2} .
\end{aligned}
$$

(III) $\mathscr{T}:=\{T(t): C \rightarrow C, t \geq 0\}$ is said to be asymptotically strict pseudocontractive semigroup, if the conditions (a) $-(\mathrm{c})$ and the following condition (f) are satisfied.

(f) There exist a bounded function $\eta:[0, \infty) \rightarrow[0, \infty)$ and a sequence $k_{n} \subset[1, \infty)$ with $k_{n} \rightarrow 1$ as $n \rightarrow \infty$. For any given $x, y \in C$, there exists $j(x-y) \in J(x-y)$ such that for, any $t \geq 0$,

$$
\begin{aligned}
& \left\langle T^{n}(t) x-T^{n}(t) y, j(x-y)\right\rangle \\
& \quad \leq k_{n}\|x-y\|^{2}-\eta(t)\left\|(x-y)-\left(T^{n}(t) x-T^{n}(t) y\right)\right\|^{2} .
\end{aligned}
$$

Osilike and Akuchu [2] established an iterative scheme for approximation of common fixed points of a finite family of asymptotically pseudocontractive mappings. Miao et al. [3] introduced an implicit iteration process for a finite family of total asymptotically pseudocontractive maps. And in recent years, many researchers focused on the convergence of pseudocontractive and asymptotically strict pseudocontractive semigroups; see [4-8] and their references. In $[9,10]$ especially, the authors gave the modified Mann type iteration algorithm and studied its convergence.

Inspired and motivated by the above works, in this paper, we give a new modified Ishikawa type iteration algorithm for total asymptotically strict pseudocontractive semigroups. Under the reducation of some conditions, we prove both strong convergence and weak convergence of the iteration algorithm by using the method of the subsequence of a subsequence of the sequence $\left\{x_{n}\right\}$ in Banach spaces, respectively. The results presented in this paper extend and improve the corresponding recent results of many authors, such as [1,7$10]$.

\section{Preliminaries}

This section contains some definitions, notations, and lemmas, which will be used in the proofs of our main results in the next section.

A Banach space $E$ is said to be smooth if the limit $\lim _{t \rightarrow 0}((\|x+t y\|-\|x\|) / t)$ exists for each $x, y \in\{x \in E$ : $\|x\|=1\}$. It is well known that if $E$ is reflexive and smooth, then the duality mapping $J$ is single valued.

A Banach space $E$ is said to have Opial condition if, for any sequence $\left\{x_{n}\right\} \subset E$ weakly convergent to $x_{0} \in E$,

$$
\liminf _{n \rightarrow \infty}\left\|x_{n}-x_{0}\right\|<\liminf _{n \rightarrow \infty}\left\|x_{n}-x\right\|
$$

holds for any $x \neq x_{0}$.

A mapping $T$ is said to be demiclosed, if, for any sequence $\left\{x_{n}\right\} \subset E, x_{n} \rightarrow y$ and $\left\|(I-T) x_{n}\right\| \rightarrow 0$ imply that $(I-T) y=$ 0 .

Definition 3 (see [9]). One-parameter $\mathscr{T}:=\{T(t): C \rightarrow C$, $t \geq 0\}$ is said to be a $\left(\eta,\left\{\mu_{n}\right\},\left\{\xi_{n}\right\}, \phi\right)$-total asymptotically strict pseudocontractive semigroup on $C$, if the conditions (a)-(c) in Definition 2 and the following condition (g) are satisfied.

(g) There exist a bounded function $\eta:[0, \infty) \rightarrow[0, \infty)$ and sequences $\left\{\mu_{n}\right\} \subset[0, \infty)$ and $\left\{\xi_{n}\right\} \subset[0, \infty)$ with $\mu_{n} \rightarrow 0, \xi_{n} \rightarrow 0$, as $n \rightarrow \infty$. For any given $x, y \in C$, there exists $j(x-y) \in J(x-y)$, such that

$$
\begin{aligned}
\left\langle T^{n}(t)\right. & \left.x-T^{n}(t) y, j(x-y)\right\rangle \\
\leq & \|x-y\|^{2}-\eta(t)\left\|(x-y)-\left(T^{n}(t) x-T^{n}(t) y\right)\right\|^{2} \\
& +\mu_{n} \phi(\|x-y\|)+\xi_{n},
\end{aligned}
$$

for any $t \geq 0$, for all $n \geq 1$, where $\phi:[0, \infty) \rightarrow$ $[0, \infty)$ is continuous and strictly increasing function with $\phi(0)=0$.

A $\left(\eta,\left\{\mu_{n}\right\},\left\{\xi_{n}\right\}, \phi\right)$-total asymptotically strict pseudocontractive semigroup is said to be uniformly Lipschitzian, if there exists a bounded measurable function $L:[0, \infty) \rightarrow$ $(0, \infty)$, such that

$$
\begin{aligned}
& \left\|T^{n}(t) x-T^{n}(t) y\right\| \\
& \quad \leq L(t)\|x-y\|, \quad \forall x, y \in C, t \geq 0, n \in \mathbb{N} .
\end{aligned}
$$


Remark 4. According to the definitions, it is obvious that a pseudocontractive semigroup is a strict pseudocontractive semigroup with $\eta(t)=0$, and a strict pseudocontractive semigroup is an asymptotically strict pseudocontractive semigroup with $k_{n}=1$. An asymptotically strict pseudocontractive semigroup is a $\left(\eta,\left\{\mu_{n}\right\},\left\{\xi_{n}\right\}, \phi\right)$-total asymptotically strict pseudocontractive semigroup with $\phi(t)=t^{2}, \mu_{n}=k_{n}-1$, and $\xi_{n}=0$.

Definition 5 (see [11]). The normalized duality mapping $J$ of a Banach space $E$ is said to be weakly sequential continuous; if for all $\left\{x_{n}\right\} \subset E, x_{n} \rightarrow x$, then there exist $j\left(x_{n}\right) \in J\left(x_{n}\right), j(x) \in$ $J(x)$ such that $j\left(x_{n}\right) \dot{-} j(x)$, where weak convergence and weak star convergence are denoted by $\rightarrow$ and $\dot{\vec{c}}$, respectively.

In order to prove the main results of this paper, the following lemmas should be used.

Lemma 6 (see [4]). For any $x, y \in E$, one has

$$
\begin{array}{r}
\|x+y\|^{2} \leq\|x\|^{2}+2\langle y, j(x-y)\rangle, \\
\forall j(x-y) \in J(x-y) .
\end{array}
$$

Lemma 7 (see [12]). Let $\left\{a_{n}\right\},\left\{b_{n}\right\}$, and $\left\{\delta_{n}\right\}$ be the sequences of $\mathbb{R}^{+}$, which satisfy

$$
a_{n+1} \leq\left(1+\delta_{n}\right) a_{n}+b_{n}, \quad \forall n \geq 1 .
$$

If $\sum_{n=1}^{\infty} \delta_{n}<\infty, \sum_{n=1}^{\infty} b_{n}<\infty$, then the limit $\lim _{n \rightarrow \infty} a_{n}$ exists.

\section{Main Results}

Theorem 8. Let $C$ be a nonempty closed convex subset of a real Banach space $E$, and let $\mathscr{T}:=\{T(t): C \rightarrow C, t \geq 0\}$ be a uniformly Lipschitzian and $\left(\eta,\left\{\mu_{n}\right\},\left\{\xi_{n}\right\}, \phi\right)$-total asymptotically strict pseudocontractive semigroup defined in Definition 3. Suppose that $F(\mathscr{T}):=\bigcap_{t \geq 0} F(T(t)) \neq \emptyset$ and there exists a compact subset $K$ of $E$ such that $\bigcap_{t \geq 0} T(t)(C) \subseteq K$. We assume that there exist positive constants $M$ and $M^{*}$, such that $\phi(x) \leq M^{*} x^{2}$ for all $x \geq M$. Let $\left\{x_{n}\right\}$ be the sequence defined by the modified Ishikawa type iteration algorithm:

$$
\begin{gathered}
x_{1} \in C, \quad \text { chosen arbitrarily, } \\
y_{n}=\left(1-\beta_{n}\right) x_{n}+\beta_{n} T^{n}(t) x_{n}, \\
x_{n+1}=\left(1-\alpha_{n}\right) x_{n}+\alpha_{n} T^{n}(t) y_{n} .
\end{gathered}
$$

Then $\left\{x_{n}\right\}$ converges strongly to a common fixed point $x^{*} \epsilon$ $F(\mathscr{T})$ in $C$, if the following conditions are satisfied:

(i) $\sum_{n=1}^{\infty} \alpha_{n}^{2}<\infty, \sum_{n=1}^{\infty} \alpha_{n}=\infty, \sum_{n=1}^{\infty} \alpha_{n} \mu_{n}<\infty$, and $\sum_{n=1}^{\infty} \alpha_{n} \xi_{n}<\infty$;

(ii) $\beta_{n} \rightarrow 0$ as $n \rightarrow \infty, \sum_{n=1}^{\infty} \alpha_{n} \beta_{n}<\infty$;

(iii) $\eta=\inf _{t \geq 0} \eta(t)>0, L=\sup _{t \geq 0} L(t)<+\infty$.

Proof. We divide the proof into four steps.

Step 1. Firstly, we prove that $\lim _{n \rightarrow \infty}\|x-p\|$ exists for any $p \in F(\mathscr{T})$.
By the definitions of $T(t)$ and $\left\{x_{n}\right\}$, we have

$$
\begin{gathered}
\left\|T^{n}(t) x_{n}-p\right\| \leq L\left\|x_{n}-p\right\|, \\
\left\|y_{n}-p\right\|=\left\|\left(1-\beta_{n}\right) x_{n}+\beta_{n} T^{n}(t) x_{n}-p\right\| \\
\leq\left(1-\beta_{n}\right)\left\|x_{n}-p\right\|+\beta_{n}\left\|T^{n}(t) x_{n}-p\right\| \\
\leq\left(1-\beta_{n}+\beta_{n} L\right)\left\|x_{n}-p\right\| \\
\leq(1+L)\left\|x_{n}-p\right\|, \\
\left\|T^{n}(t) y_{n}-p\right\| \leq L\left\|y_{n}-p\right\| \leq L(1+L)\left\|x_{n}+p\right\| .
\end{gathered}
$$

This follows from that

$$
\begin{aligned}
\left\|x_{n+1}-p\right\| & =\left\|\left(1-\alpha_{n}\right) x_{n}+\alpha_{n} T^{n}(t) y_{n}-p\right\| \\
& \leq\left(1-\alpha_{n}\right)\left\|x_{n}-p\right\|+\alpha_{n}\left\|T^{n}(t) y_{n}-p\right\| \\
& \leq\left(1-\alpha_{n}\right)\left\|x_{n}-p\right\|+\alpha_{n} L(1+L)\left\|x_{n}-p\right\| \\
& \leq\left(1+L+L^{2}\right)\left\|x_{n}-p\right\|, \\
\left\|y_{n}-x_{n}\right\| & =\left\|\left(1-\beta_{n}\right) x_{n}+\beta_{n} T^{n}(t) x_{n}-x_{n}\right\| \\
& =\beta_{n}\left\|x_{n}-T^{n}(t) x_{n}\right\| \\
& \leq \beta_{n}\left(\left\|x_{n}-p\right\|+\left\|T^{n}(t) x_{n}-p\right\|\right) \\
& \leq \beta_{n}(1+L)\left\|x_{n}-p\right\|, \\
\left\|T^{n}(t) y_{n}-x_{n}\right\| & \leq\left\|T^{n}(t) y_{n}-p\right\|+\left\|x_{n}-p\right\| \\
& \leq\left(1+L+L^{2}\right)\left\|x_{n}-p\right\|, \\
\left\|x_{n+1}-x_{n}\right\| & =\left\|\left(1-\alpha_{n}\right) x_{n}+\alpha_{n} T^{n}(t) y_{n}-x_{n}\right\| \\
& \leq \alpha_{n}\left\|T^{n}(t) y_{n}-x_{n}\right\| \\
& \leq \alpha_{n}\left(1+L+L^{2}\right)\left\|x_{n}-p\right\| .
\end{aligned}
$$

Since $\mathscr{T}:=\{T(t): C \rightarrow C, t \geq 0\}$ is total asymptotically strict pseudocontractive semigroup, for any point $x_{n+1} \in C$ and $p \in F(\mathscr{T})$, by (9), we have

$$
\begin{aligned}
& \left\langle T^{n}(t) x_{n+1}-x_{n+1}, j\left(x_{n+1}-p\right)\right\rangle \\
& \quad \leq-\eta(t)\left\|T^{n}(t) x_{n+1}-x_{n+1}\right\|+\mu_{n} \phi\left(\left\|x_{n+1}-p\right\|\right)+\xi_{n} .
\end{aligned}
$$

Since $\phi$ is an increasing function, it results in that $\phi(x) \leq$ $\phi(M)$, if $x \leq M ; \phi(x) \leq M^{*} x^{2}$, if $x \geq M$. In either case, we can obtain that

$$
\phi(x) \leq \phi(M)+M^{*} x^{2} .
$$


Hence, by Lemma 6, we have

$$
\begin{aligned}
\| x_{n+1}- & p \|^{2} \\
= & \left\|x_{n}-p+\alpha_{n}\left(T^{n}(t) y_{n}-x_{n}\right)\right\|^{2} \\
\leq & \left\|x_{n}-p\right\|^{2}+2 \alpha_{n}\left\langle T^{n}(t) y_{n}-T^{n}(t) x_{n}, j\left(x_{n+1}-p\right)\right\rangle \\
& +2 \alpha_{n}\left\langle T^{n}(t) x_{n}-T^{n}(t) x_{n+1}, j\left(x_{n+1}-p\right)\right\rangle \\
& +2 \alpha_{n}\left\langle T^{n}(t) x_{n+1}-x_{n+1}, j\left(x_{n+1}-p\right)\right\rangle \\
& +2 \alpha_{n}\left\langle x_{n+1}-x_{n}, j\left(x_{n+1}-p\right)\right\rangle \\
\leq & \left\|x_{n}-p\right\|^{2}+2 \alpha_{n} L\left\|y_{n}-x_{n}\right\| \cdot\left\|x_{n+1}-p\right\| \\
& +2 \alpha_{n}\left\|x_{n}-x_{n+1}\right\| \cdot\left\|x_{n+1}-p\right\|^{2} \\
& -2 \alpha_{n} \eta(t)\left\|T^{n}(t) x_{n+1}-x_{n+1}\right\|^{2} \\
& +2 \alpha_{n}\left\|x_{n}-x_{n+1}\right\| \cdot\left\|x_{n+1}-p\right\|^{2} \\
& +2 \alpha_{n} \mu_{n} \phi\left(\left\|x_{n+1}-p\right\|\right)+2 \alpha_{n} \xi_{n} \\
\leq & \left\|x_{n}-p\right\|^{2}+2 \alpha_{n} \beta_{n} L(1+L)\left(1+L+L^{2}\right)\left\|x_{n}-p\right\|^{2} \\
& +2 \alpha_{n}^{2}\left(1+L+L^{2}\right)^{2}\left\|x_{n}-p\right\|^{2} \\
& -2 \alpha_{n} \eta(t)\left\|T^{n}(t) x_{n+1}-x_{n+1}\right\|^{2} \\
& +2 \alpha_{n}^{2}\left(1+L+L^{2}\right)^{2}\left\|x_{n}-p\right\|^{2} \\
& +2 \alpha_{n} \mu_{n}\left[\phi(M)+M^{*}\left(1+L+L^{2}\right)\left\|x_{n}-p\right\|^{2}\right] \\
& \left(1+\alpha_{n} \xi_{n}\right. \\
& \left.+\delta_{n}\right)\left\|x_{n}-p\right\|^{2}+b_{n}, \\
& \\
&
\end{aligned}
$$

where $\delta_{n}=\left[2 \alpha_{n} \beta_{n} L(1+L)+4 \alpha_{n}^{2}\left(1+L+L^{2}\right)+2 M^{*} \alpha_{n} \mu_{n}\right]$ $\left(1+L+L^{2}\right), b_{n}=2 \alpha_{n} \mu_{n} \phi(M)+2 \alpha_{n} \xi_{n}$.

By the conditions (i) and (ii), we have $\sum_{n=1}^{\infty} \delta_{n}<\infty$, $\sum_{n=1}^{\infty} b_{n}<\infty$. Thus, by Lemma 7 , we can obtain that $\lim _{n \rightarrow \infty}\left\|x_{n}-p\right\|$ exists.

Step 2. Now we prove that $\liminf _{n \rightarrow \infty}\left\|x_{n}-T^{n}(t) x_{n}\right\|=0$.

From (18), we know that

$$
\begin{aligned}
& 2 \alpha_{n} \eta(t)\left\|T^{n}(t) x_{n+1}-x_{n+1}\right\|^{2} \\
& \quad \leq\left(\left\|x_{n}-p\right\|^{2}-\left\|x_{n+1}-p\right\|^{2}\right)+\delta_{n}\left\|x_{n}-p\right\|^{2}+b_{n} .
\end{aligned}
$$

As $\eta=\inf _{t \geq 0} \eta(t)>0, A=\sup _{n}\left\|x_{n}-p\right\|<\infty$, we can have

$$
\begin{aligned}
& \sum_{n=1}^{m} 2 \alpha_{n} \eta\left\|T^{n}(t) x_{n+1}-x_{n+1}\right\|^{2} \\
& \quad \leq \sum_{n=1}^{m}\left[\left(\left\|x_{n}-p\right\|^{2}-\left\|x_{n+1}-p\right\|^{2}\right)+\delta_{n} A^{2}+b_{n}\right] \\
& \leq\left\|x_{1}-p\right\|^{2}+A^{2} \sum_{n=1}^{m} \delta_{n}+\sum_{n=1}^{m} b_{n} .
\end{aligned}
$$

Then,

$$
\begin{aligned}
& \sum_{n=1}^{\infty} 2 \alpha_{n} \eta\left\|T^{n}(t) x_{n+1}-x_{n+1}\right\|^{2} \\
& \quad \leq \sum_{n=1}^{\infty}\left[\left(\left\|x_{n}-p\right\|^{2}-\left\|x_{n+1}-p\right\|^{2}\right)+\delta_{n} A^{2}+b_{n}\right] \\
& \leq\left\|x_{1}-p\right\|^{2}+A^{2} \sum_{n=1}^{\infty} \delta_{n}+\sum_{n=1}^{\infty} b_{n}<\infty .
\end{aligned}
$$

Since $\sum_{n=1}^{\infty} \alpha_{n}=\infty$, then (21) implies that

$$
\liminf _{n \rightarrow \infty}\left\|x_{n+1}-T^{n}(t) x_{n+1}\right\|=0
$$

Otherwise, if liminf $\operatorname{in}_{n \rightarrow \infty}\left\|x_{n+1}-T^{n}(t) x_{n+1}\right\|=c>0$, then there exists an $N$, such that $\left\|x_{n}-T^{n}(t) x_{n}\right\| \geq c / 2$, when $n \geq N$. So, we have

$$
\begin{aligned}
\sum_{n=1}^{\infty} 2 \alpha_{n} \eta\left\|T^{n}(t) x_{n+1}-x_{n+1}\right\|^{2} & \\
= & \sum_{n=1}^{N} 2 \alpha_{n} \eta\left\|T^{n}(t) x_{n+1}-x_{n+1}\right\|^{2} \\
& +\sum_{n=N}^{\infty} 2 \alpha_{n} \eta\left\|T^{n}(t) x_{n+1}-x_{n+1}\right\|^{2} \\
\geq & \sum_{n=1}^{N} 2 \alpha_{n} \eta\left\|T^{n}(t) x_{n+1}-x_{n+1}\right\|^{2}+\frac{c^{2}}{2 \eta} \sum_{n=N}^{\infty} \alpha_{n}=\infty
\end{aligned}
$$

This is in contradiction with (21).

Because

$$
\begin{aligned}
\| x_{n}- & T^{n}(t) x_{n} \| \\
\leq & \left\|x_{n}-x_{n+1}\right\|+\left\|x_{n+1}-T^{n}(t) x_{n+1}\right\| \\
& +\left\|T^{n}(t) x_{n+1}-T^{n}(t) x_{n}\right\| \\
\leq & \left\|x_{n+1}-T^{n}(t) x_{n+1}\right\|+(1+L)\left\|x_{n}-x_{n+1}\right\| \\
\leq & \left\|x_{n+1}-T^{n}(t) x_{n+1}\right\| \\
& +\alpha_{n}(1+L)\left(1+L+L^{2}\right) A
\end{aligned}
$$

and $\lim _{n \rightarrow \infty} \alpha_{n}=0$, we have

$$
\liminf _{n \rightarrow \infty}\left\|x_{n}-T^{n}(t) x_{n}\right\|=0 \text {. }
$$

Step 3. Now we prove that $\liminf _{n \rightarrow \infty}\left\|x_{n}-T(t) x_{n}\right\|=0$. 
Consider

$$
\begin{aligned}
\| x_{n+1}- & T(t) x_{n+1} \| \\
\leq & \left\|x_{n+1}-T^{n+1}(t) x_{n+1}\right\| \\
& +\left\|T^{n+1}(t) x_{n+1}-T(t) x_{n+1}\right\| \\
\leq & \left\|x_{n+1}-T^{n+1}(t) x_{n+1}\right\| \\
& +L\left\|T^{n}(t) x_{n+1}-x_{n+1}\right\| \\
\leq & \left\|x_{n+1}-T^{n+1}(t) x_{n+1}\right\| \\
& +L\left(\left\|T^{n}(t) x_{n}-T^{n}(t) x_{n}\right\|\right. \\
& \left.\quad+\left\|T^{n}(t) x_{n}-x_{n}\right\|+\left\|x_{n}-x_{n+1}\right\|\right) \\
\leq & \left\|x_{n+1}-T^{n+1}(t) x_{n+1}\right\|+L\left\|T^{n}(t) x_{n}-x_{n}\right\| \\
& +L(1+L)\left\|x_{n}-x_{n+1}\right\| \\
\leq & \left\|x_{n+1}-T^{n+1}(t) x_{n+1}\right\|+L\left\|T^{n}(t) x_{n}-x_{n}\right\| \\
& +\alpha_{n} L(1+L)\left(1+L+L^{2}\right) A .
\end{aligned}
$$

Since $\lim _{n \rightarrow \infty} \alpha_{n}=0$ and (25), we have

$$
\liminf _{n \rightarrow \infty}\left\|x_{n}-T(t) x_{n}\right\|=0 \text {. }
$$

Thus, there exists a subsequence $\left\{x_{n_{k}}\right\} \subseteq\left\{x_{n}\right\}$ such that

$$
\lim _{n \rightarrow \infty}\left\|x_{n_{k}}-T(t) x_{n_{k}}\right\|=0 .
$$

Step 4. Finally, we prove the sequence $\left\{x_{n}\right\}$ converges strongly to a common fixed point of the semigroup $\mathscr{T}:=\{T(t): C \rightarrow$ $C, t \geq 0\}$.

Since $K$ is a compact subset of $E$ and $\bigcap_{t \geq 0} T(t)(C) \subseteq K$, just as the proof in $[9,10]$, there exists a subsequence $\left\{x_{n_{k_{i}}}\right\} \subseteq$ $\left\{x_{n_{k}}\right\} \subseteq\left\{x_{n}\right\} \subseteq C$, such that $T(t) x_{n_{k_{i}}} \rightarrow x^{*} \in K$. From (28), we have $\lim _{n_{k_{i}} \rightarrow \infty}\left\|T(t) x_{n_{k_{i}}}-x_{n_{k_{i}}}\right\|=0$, and

$$
\left\|x_{n_{k_{i}}}-x^{*}\right\| \leq\left\|x_{n_{k_{i}}}-T(t) x_{n_{k_{i}}}\right\|+\left\|T(t) x_{n_{k_{i}}}-x^{*}\right\| \longrightarrow 0 .
$$

Hence we have that

$$
\left\|T(t) x^{*}-x^{*}\right\|=\lim _{n_{k_{i}} \rightarrow \infty}\left\|x_{n_{k_{i}}}-T(t) x_{n_{k_{i}}}\right\|=0 .
$$

That is, $x^{*} \in F(\mathscr{T})$.

Since, for any $p \in F(\mathscr{T}), \lim _{n \rightarrow \infty}\left\|x_{n}-p\right\|$ exists, $\lim _{n_{k_{i}} \rightarrow \infty}\left\|x_{n_{k_{i}}}-x^{*}\right\|=0$, and $\left\{x_{n_{k_{i}}}\right\} \subseteq\left\{x_{n}\right\}$, so we have $\lim _{n \rightarrow \infty}\left\|x_{n}-p\right\|=0$; that is, $x_{n}$ converges strongly to an element $x^{*}=p$ of $F(\mathscr{T})$.

Remark 9. (a) If we take $\beta_{n}=0$ in the modified Ishikawa type iteration algorithm (13), then (13) is called the modified Mann type iteration algorithm in many articles, such as in $[9,10]$. (b) In Theorem 8 , because there is no limit to $t$ of $T(t)$, so our result is stronger and the conditions here are less than in $[9,10]$. For example, the conditions "for any bounded subset $D \subset C$,

$$
\lim _{n \rightarrow \infty} \sup _{x \in D, s \in \mathbb{R}^{+}}\left\|T^{n}\left(s+t_{n}\right) x-T^{n}\left(t_{n}\right) x\right\|=0 ”
$$

in $[9,10]$ can be removed in Theorem 8 . (c) The condition "there exists a compact subset $K$ of $E$ such that $\bigcap_{t \geq 0} T(t)(C) \subseteq$ $K$ " does not look natural. But it easy to see that this condition is established naturally when we assume $C$ is a compact subset of $E$. So, the result in Theorem 8 is still true if this condition is replaced by the condition "let $C$ be a compact subset of $E$." If there is no compactness assumption, we can get the following weak convergence theorem.

Theorem 10. Let $E$ be a reflexive Banach space satisfying the opial condition and $C$ be a nonempty bounded closed convex subset of $E$. Let $\mathscr{T}:=\{T(t): C \rightarrow C, t \geq 0\}$ be a uniformly Lipschitzian and $\left(\eta,\left\{\mu_{n}\right\},\left\{\xi_{n}\right\}, \phi\right)$-total asymptotically strict pseudocontractive semigroup defined by Definition 3. Suppose that there exist positive constants $M$ and $M^{*}$, such that $\phi(x) \leq$ $M^{*} x^{2}$, for all $x \geq M$, and $F(\mathscr{T}):=\bigcap_{t \geq 0} F(T(t)) \neq \emptyset$. Let $\left\{x_{n}\right\}$ be the sequence defined by (13). Then $\left\{x_{n}\right\}$ converges weakly to a common fixed point $x^{*} \in F(\mathscr{T})$ in $C$, if the following conditions are satisfied.

(i) $\sum_{n=1}^{\infty} \alpha_{n}^{2}<\infty, \sum_{n=1}^{\infty} \alpha_{n}=\infty, \sum_{n=1}^{\infty} \alpha_{n} \mu_{n}<\infty$,
$\sum_{n=1}^{\infty} \alpha_{n} \xi_{n}<\infty$.

(ii) $\beta_{n} \rightarrow 0$ as $n \rightarrow \infty$, and $\sum_{n=1}^{\infty} \alpha_{n} \beta_{n}<\infty$.

(iii) $\lambda=\inf _{t \geq 0} \lambda(t)>0, L=\sup _{t \geq 0} L(t)<+\infty$.

Proof. It can be proved just like the proof in Theorem 8 that, for each $p \in F(\mathscr{T}), \lim _{n \rightarrow \infty}\left\|x_{n}-p\right\|$ exists, and, for all $t>0$, $T(t) x_{n}$ is bounded, liminf $\left\|T(t) x_{n}-x_{n}\right\|=0$. Thus, there exists a subsequence $\left\{x_{n_{k}}\right\} \subseteq\left\{x_{n}\right\}$ such that $\lim _{n \rightarrow \infty} \| x_{n_{k}}-$ $T(t) x_{n_{k}} \|=0$.

Now we prove that $I-T(t)$ is demiclosed at zero (see [11]).

Since $C$ is a closed and convex subset of a reflexive Banach space $E$, there exists a subsequence $\left\{x_{n_{k_{i}}}\right\} \subseteq\left\{x_{n_{k}}\right\} \subseteq\left\{x_{n}\right\}$, such that $x_{n_{k_{i}}} \rightarrow x^{*} \in C$. Without loss of generality, we can assume that $\left\{x_{n}\right\}$ replaces $\left\{x_{n_{k_{i}}}\right\}$ now.

In the following, we prove that $x^{*}=T(t) x^{*}$.

Firstly, we choose $\alpha \in(0,1 /(1+L))$ and $y_{m}=(1-\alpha) x+$ $T^{m}(t) x$ for $m \geq 1$. Since $T(t)$ is uniformly Lipschitzian, we have

$$
\begin{aligned}
\left\|x_{n}-T^{m}(t) x_{n}\right\| & \leq \sum_{k=0}^{m-1}\left\|T^{k}(t) x_{n}-T^{k+1}(t) x_{n}\right\| \\
& \leq \sum_{k=0}^{m-1} L\left\|x_{n}-T(t) x_{n}\right\| \\
& =m L\left\|x_{n}-T(t) x_{n}\right\| \longrightarrow 0, \quad n \longrightarrow \infty .
\end{aligned}
$$


Because $T(t)$ is totally asymptotically strictly pseudocontractive, we have

$$
\begin{aligned}
&\left\langle\left(I-T^{m}(t)\right) y_{m}, J\left(x-y_{m}\right)\right\rangle \\
&=\left\langle\left(I-T^{m}(t)\right) y_{m}, J\left(x-y_{m}\right)-J\left(x_{n}-y_{m}\right)\right\rangle \\
&+\left\langle\left(I-T^{m}(t)\right) y_{m}, J\left(x_{n}-y_{m}\right)\right\rangle \\
&=\left\langle\left(I-T^{m}(t)\right) y_{m}, J\left(x-y_{m}\right)-J\left(x_{n}-y_{m}\right)\right\rangle \\
&+\left\langle\left(I-T^{m}(t)\right) x_{n}, J\left(x_{n}-y_{m}\right)\right\rangle \\
&+\left\langle\left(I-T^{m}(t)\right) y_{m}-\left(I-T^{m}(t)\right) x_{n}, J\left(x_{n}-y_{m}\right)\right\rangle \\
&=\left\langle\left(I-T^{m}(t)\right) y_{m}, J\left(x-y_{m}\right)-J\left(x_{n}-y_{m}\right)\right\rangle \\
&+\left\langle\left(I-T^{m}(t)\right) x_{n}, J\left(x_{n}-y_{m}\right)\right\rangle \\
&-\eta(t)\left\|\left(I-T^{m}(t)\right) x_{n}-\left(I-T^{m}(t)\right) y_{m}\right\|^{2} \\
&+\mu_{n} \phi\left(\left\|x_{n}-y_{n}\right\|\right)+\xi_{n} \\
& \leq\left\langle\left(I-T^{m}(t)\right) y_{m}, J\left(x-y_{m}\right)-J\left(x_{n}-y_{m}\right)\right\rangle \\
&+\left\langle\left(I-T^{m}(t)\right) x_{n}, J\left(x_{n}-y_{n}\right)\right\rangle \\
&+\mu_{n}\left(M+M^{*}\left\|x_{n}-y_{m}\right\|^{2}\right)+\xi_{n} .
\end{aligned}
$$

Since $x_{n} \rightarrow x^{*}, \lim _{n \rightarrow \infty}\left\|x_{n}-T(t) x_{n}\right\|=0$ (note the $x_{n}$ of here instead of $x_{n_{k_{i}}}$ ), and $J$ is weakly sequential continuous duality mapping, we have

$$
\begin{aligned}
\langle(I- & \left.\left.T^{m}(t)\right) x^{*}-\left(I-T^{m}(t)\right) y_{m}, J\left(x^{*}-y_{m}\right)\right\rangle \\
& \leq(1+L)\left\|x^{*}-y_{m}\right\|^{2} \\
& \leq(1+L) \alpha^{2}\left\|x^{*}-T^{m}(t) x^{*}\right\|^{2} .
\end{aligned}
$$

Hence

$$
\begin{aligned}
\| x^{*}- & T^{m}(t) x^{*} \|^{2} \\
= & \left\langle x^{*}-T^{m}(t) x^{*}, J\left(x^{*}-T^{m}(t) x^{*}\right)\right\rangle \\
= & \frac{1}{\alpha}\left\langle x^{*}-T^{m}(t) x^{*}, J\left(x^{*}-y_{m}\right)\right\rangle \\
= & \frac{1}{\alpha}\left\langle x^{*}-T^{m}(t) x^{*}-\left(y_{m}-T^{m}(t) y_{m}\right), J\left(x^{*}-y_{m}\right)\right\rangle \\
& +\frac{1}{\alpha}\left\langle y_{m}-T^{m}(t) y_{m}, J\left(x^{*}-y_{m}\right)\right\rangle \\
\leq & \alpha(1+L)\left\|x^{*}-T^{m}(t) x^{*}\right\|^{2} \\
& +\frac{1}{\alpha}\left(\mu_{m} M+\mu_{m} M^{*}(\operatorname{diam} C)+\xi_{n}\right) .
\end{aligned}
$$

This implies that

$$
\begin{aligned}
& \alpha(1-\alpha(1+L))\left\|x^{*}-T^{m}(t) x^{*}\right\|^{2} \\
& \quad \leq \mu_{m} M+\mu_{m} M^{*}(\operatorname{diam} C)+\xi_{n}, \quad \forall m \in \mathbb{N} .
\end{aligned}
$$

Let $m \rightarrow \infty$; then we have $\left\|x^{*}-T^{m}(t) x^{*}\right\| \rightarrow 0$, as $m \rightarrow \infty$, for $\mu_{n} \rightarrow 0, \xi_{n} \rightarrow 0$. Hence, $T^{m}(t) x^{*} \rightarrow x^{*}$, as $m \rightarrow \infty$, and $T^{m+1}(t) x^{*} \rightarrow T(t) x^{*}$. By the continuity of $T(t)$, we have $T(t) x^{*}=x^{*}$.

Now, for the sequence $\left\{x_{n}\right\}$ generated by (13), we prove that $x_{n} \rightarrow x^{*}$.

Suppose the contrary; if there exists another subsequence $\left\{x_{n_{j}}\right\} \subset\left\{x_{n}\right\}$, such that $x_{n_{j}} \rightarrow y^{*}$ with $y^{*} \neq x^{*}$, then we have that $\lim _{n \rightarrow \infty}\left\|x_{n}-x^{*}\right\|$ and $\lim _{n \rightarrow \infty}\left\|x_{n}-y^{*}\right\|$ exist. Since $E$ satisfies the Opial condition, we have

$$
\begin{aligned}
\liminf _{n_{k_{i}} \rightarrow \infty}\left\|x_{n_{k_{i}}}-x^{*}\right\| \\
\quad<\lim _{n_{k_{i}} \rightarrow \infty}\left\|x_{n_{k_{i}}}-y^{*}\right\|=\lim _{n \rightarrow \infty}\left\|x_{n}-y^{*}\right\| \\
=\lim _{n_{j} \rightarrow \infty}\left\|x_{n_{j}}-y^{*}\right\|<\liminf _{n_{j} \rightarrow \infty}\left\|x_{n_{j}}-x^{*}\right\| \\
=\lim _{n \rightarrow \infty}\left\|x_{n}-x^{*}\right\|=\liminf _{n_{k_{i}} \rightarrow \infty}\left\|x_{n_{k_{i}}}-x^{*}\right\| .
\end{aligned}
$$

This is a contraction, which shows $x^{*}=y^{*}$. Therefore, $x_{n} \rightarrow$ $x^{*} \in F(\mathscr{T})$. This completes the proof.

Remark 11. (a) Our results extend many other results that have been proved for this important class of general pseudocontractive mappings. For example, we extend the total asymptotically strict pseudocontractive mapping in [1] and Lipschitzian pseudo-contraction semigroup in [8] to the total asymptotically strict pseudocontractive semigroup. (b) In addition, we study the weak convergence of the total asymptotically strict pseudocontractive semigroup by using the demiclosedness of $I-T(t)$ which, in some way, extends the result in [11] in Banach spaces. (c) And the method by using the subsequence of a subsequence of the sequence $\left\{x_{n}\right\}$ in this paper is different from the previous references.

\section{Acknowledgments}

The authors would like to thank editors and referees for many useful comments and suggestions for the improvement of the paper. This work was partially supported by the Natural Science Foundation of Zhejiang Province (Y6100696) and the National Natural Science Foundation of China (11271330).

\section{References}

[1] S. S. Chang, L. Wang, Y. K. Tang, and L. Yang, "The split common fixed point problem for total asymptotically strictly pseudocontractive mappings," Journal of Applied Mathematics, vol. 2012, Article ID 385638, 13 pages, 2012.

[2] M. O. Osilike and B. G. Akuchu, "Common fixed points of a finite family of asymptotically pseudocontractive maps," Fixed Point Theory and Applications, vol. 2004, no. 2, pp. 81-88, 2004.

[3] Q. Miao, R. Chen, and H. Zhou, "Convergence of an implicit iteration process for a finite family of total asymptotically pseudocontractive maps," International Journal of Mathematical Analysis, vol. 2, no. 9-12, pp. 433-436, 2008.

[4] Y. H. Wang, "Strong convergence theorems for asymptotically weak $G$-pseudo- $\Psi$-contractive non-self-mappings with 
the generalized projection in Banach spaces," Abstract and Applied Analysis, vol. 2012, Article ID 651304, 11 pages, 2012.

[5] X. Li, J. K. Kim, and N. Huang, "Viscosity approximation of common fixed points for $L$-Lipschitzian semigroup of pseudocontractive mappings in Banach spaces," Journal of Inequalities and Applications, vol. 2009, Article ID 936121, 16 pages, 2009.

[6] W. Xu and Y. H. Wang, "Strong convergence of the iterative methods for hierarchical fixed point problems of an infinite family of strictly nonself pseudocontractions," Abstract and Applied Analysis, vol. 2012, Article ID 457024, 11 pages, 2012.

[7] S. Zhang, "Convergence theorem of common fixed points for Lipschitzian pseudo-contraction semi-groups in Banach spaces," Applied Mathematics and Mechanics, vol. 30, no. 2, pp. 145-152, 2009.

[8] J. Quan, S. Chang, and M. Liu, "Strong and weak convergence of an implicit iterative process for pseudocontractive semigroups in Banach space," Fixed Point Theory and Applications, vol. 2012, article 142, 2012.

[9] L. Yang and F. H. Zhao, "Large strong convergence theorems for total asymptotically strict pseudocontractive semigroup in Banach spaces," Fixed Point Theory and Applications, vol. 2012, article 24, 2012.

[10] S. Chang, Y. J. Cho, H. W. J. Lee, and C. K. Chan, "Strong convergence theorems for Lipschitzian demicontraction semigroups in Banach spaces," Fixed Point Theory and Applications, vol. 2011, Article ID 583423, 10 pages, 2011.

[11] Y.-H. Wang and Y.-H. Xia, "Strong convergence for asymptotically pseudocontractions with the demiclosedness principle in Banach spaces," Fixed Point Theory and Applications, vol. 2012, article 45, 2012.

[12] K. Aoyama, Y. Kimura, W. Takahashi, and M. Toyoda, "Approximation of common fixed points of a countable family of nonexpansive mappings in a Banach space," Nonlinear Analysis: Theory, Methods \& Applications, vol. 67, no. 8, pp. 2350-2360, 2007. 


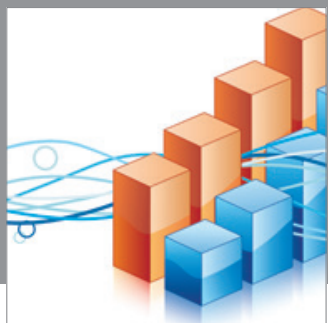

Advances in

Operations Research

mansans

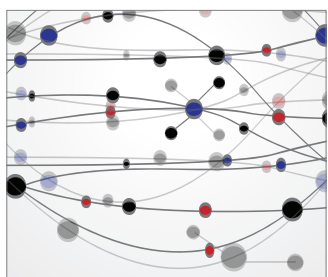

The Scientific World Journal
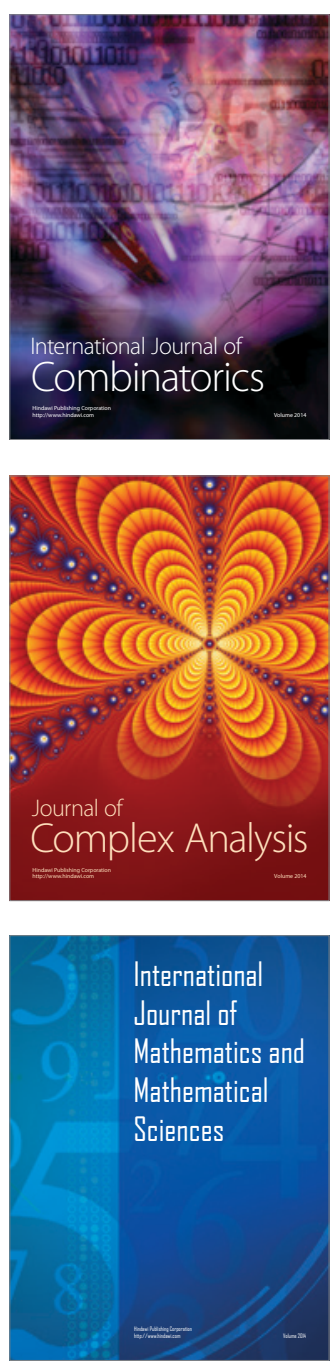
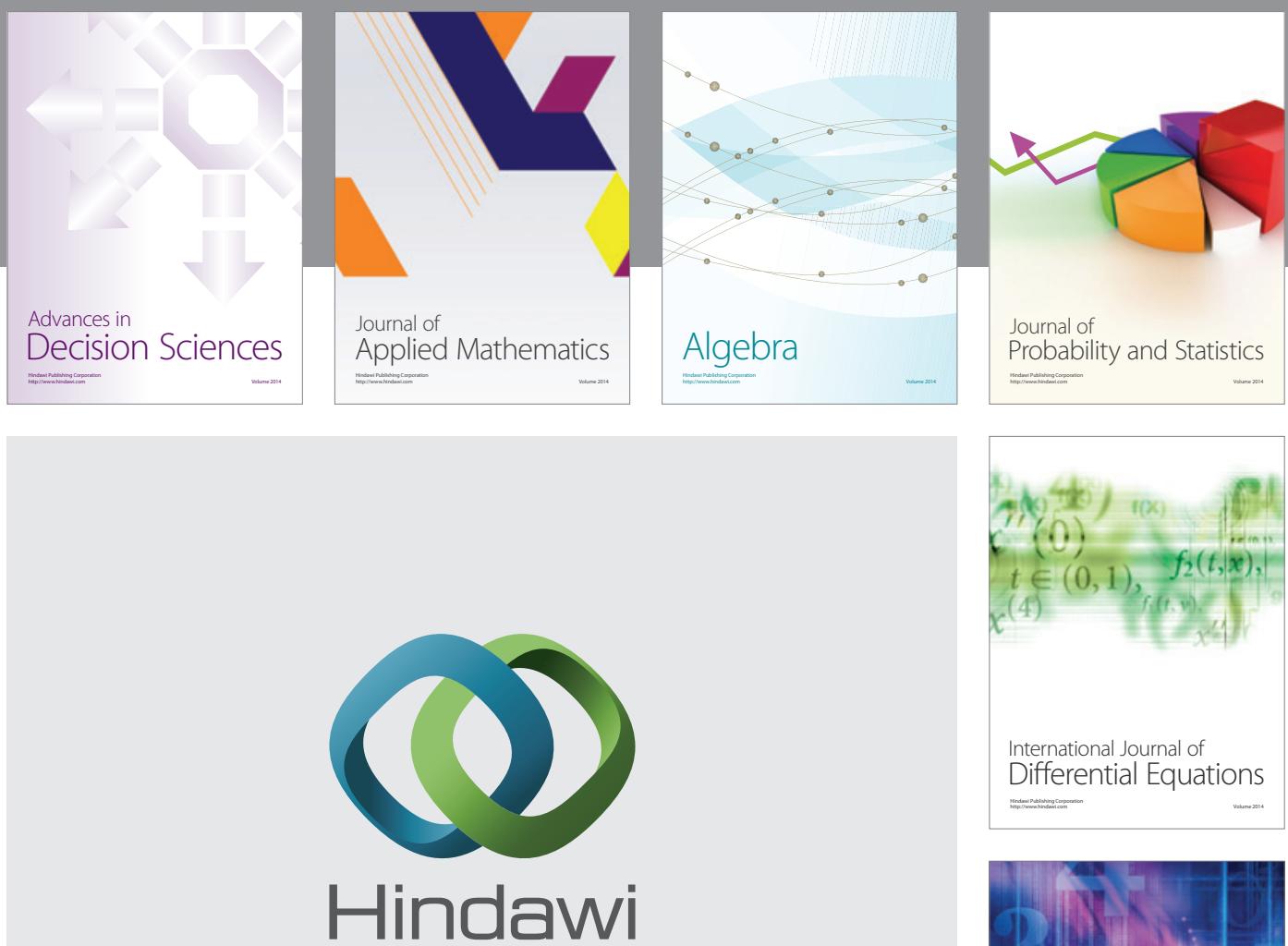

Submit your manuscripts at http://www.hindawi.com
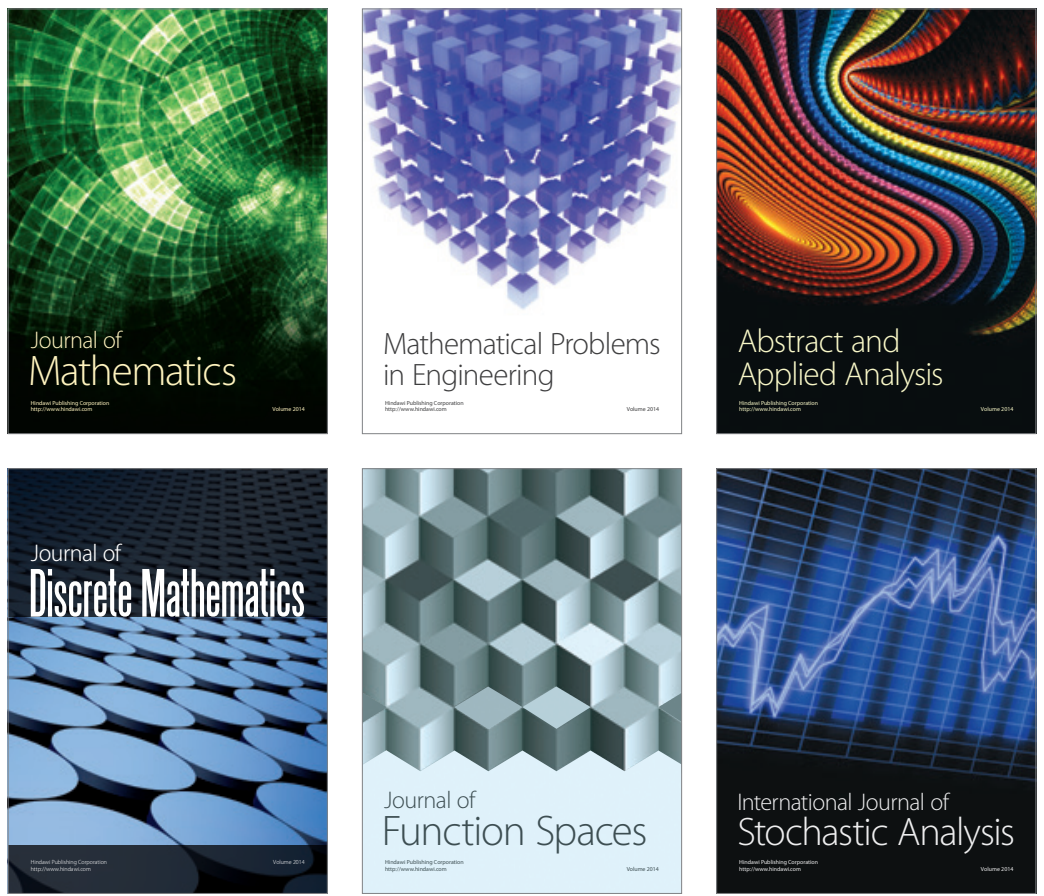

Journal of

Function Spaces

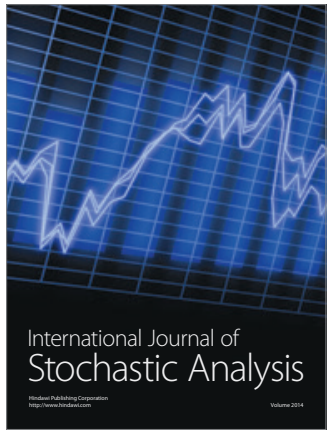

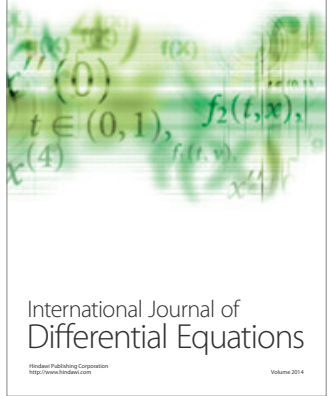
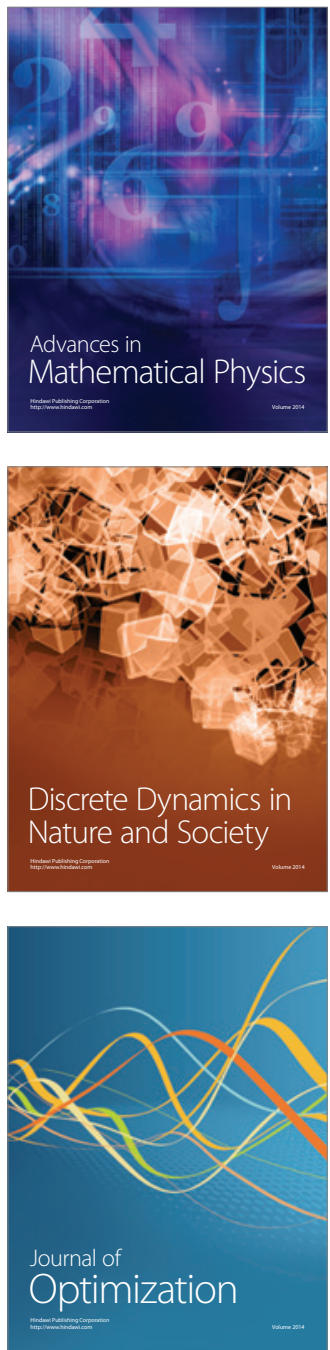